\title{
GENERAL ASPECTS OF THE THEORY OF SUMMABLE SERIES.
}

\author{
BY PROFESSOR R. D. CARMICHAEL.
}

(Read at the Chicago Symposium of the American Mathematical Society, April 12, 1918.)

\section{§1. General Considerations Relating to the Sum of an Infinite Series.}

IN 1811 Fourier* read before the Paris Academy a memoir which contained an acceptable definition of convergence of an infinite series; but this work remained unpublished for eight or nine years. In 1817 Bolzano stated a precise definition of convergence. Independently in 1821 Cauchy also formulated the definition in an exact manner. He and Abel insisted so forcefully upon the necessity of the distinction between convergence and divergence and the danger in employing divergent series that the latter came into such disrepute as not to be studied systematically for nearly three quarters of a century. For a long time no one saw how to obviate the difficulties pointed out so incisively by those who first recognized the pitfalls in the use of divergent series. And yet both Abel and Cauchy, the leading instigators, had misgivings $\dagger$ as to the justice of the decision by which these series were banished from the mathematical community and they were given up as friends who had done some things well but could not be trusted because they had also done some things ill.

Certain difficulties, however, still remain when one tries to treat convergent series independently of any reference to divergent series, as we shall show more fully in a moment.

In the first attempt to formulate a suitable definition of

* For references relating to the first paragraph see Encyclopédie des Sciences mathématiques, I, $1_{2}$, pp. 211-214.

$\dagger$ See quotations in Bromwich's Infinite Series, 1908, p. 264. Indeed Cauchy himself showed how the celebrated series of Stirling in the theory of the gamma function could be used in a legitimate way for purposes of numerical computation.

$\ddagger$ An interesting and valuable discussion of several topics in the theory of divergent series and continued fractions will be found in Van Vleck's lectures at the Boston Colloquium in 1903, published in 1905 . In these lectures some topics are treated to which we do not refer in this paper. 
the sum of the infinite series

$$
u_{0}+u_{1}+u_{2}+\cdots
$$

it is natural to employ with Cauchy the sum $s_{n}$ of the first $n+1$ terms, namely,

$$
s_{n}=u_{0}+u_{1}+\therefore+u_{n},
$$

and to say that the series (1) has the sum $s$ in case $\lim _{n=\infty} s_{n}$ exists and has the finite value $s$. But there is no good reason why we should confine attention to this definition alone when our researches so often bring us face to face with series not possessing a sum in this sense.

As a matter of fact one does not have to go far to find the inadequacy of this definition. One of the leading tasks in developing the theory of infinite series is to determine the fundamental laws of operation according to which one may compute with them. Certain of these are at once obvious, as for instance those associated with the introduction or removal of a finite number of terms, the term by term addition of two series, and the multiplication of a series term by term by a constant. But if one undertakes to form the product of two series the case is different. Consider the product of (1) by the series

$$
v_{0}+v_{1}+v_{2}+\cdots \text {. }
$$

Whenever the two series are absolutely convergent and have the sums $u$ and $v$ respectively, it may be shown without difficulty that the Cauchy product series*

where

$$
\begin{gathered}
w_{0}+w_{1}+w_{2}+\cdots, \\
w_{n}=u_{0} v_{n}+u_{1} v_{n-1}+\cdots+u_{n} v_{0}
\end{gathered}
$$

converges and has a sum $w$ which is equal to $u v$. (A like conclusion is true also under certain less restrictive hypotheses.) But the mere convergence of the $u$-series and the $v$-series does not necessitate the convergence of the $w$-series.

* This definition of product of two series is that most naturally associated with power series; but there is nothing inherently essential in it. The product of two series may in fact be defined in any one of a variety of ways, with consequent variations in the theory. On account of the importance of power series, however, it is desirable to have a theory of infinite series adequate for the case in which multiplication is defined in accordance with the Cauchy product formula. 
Starting from the problem of forming the product of two convergent series, Cesàro* in 1890 was led to an investigation of what he called indeterminate series. He showed that, when the $u$-series and $v$-series above converge to the sums $u$ and $v$ respectively, then the corresponding $w$-series has the property expressed in the relation

where

$$
\lim _{n=\infty} \frac{W_{0}+W_{1}+\cdots+W_{n}}{n+1}=u v
$$

$$
W_{n}=w_{0}+w_{1}+\cdots+w_{n} .
$$

Thus he was led to say that series (3) has the sum $w$ whenever the limit in (4) exists and has the finite value $w$. (It is easy to show-see $\$ 2$ below-that this new definition assigns to every convergent series the same sum as the usual definition.)

By the introduction of this definition the multiplication problem in which Cesàro was interested became enlarged. Suppose now that the series $u$ and $v$ have sums in the new sense; what can be said of the product series $w$ ? Following up this question, Cesàro was led to extend further the definition of sum of an infinite series. Thus when we have for a finite $s$ the relation

$$
s=\lim _{n=\infty} \frac{S_{n}{ }^{(r)}}{D_{n}{ }^{(r)}},
$$

where

$$
\begin{aligned}
S_{n}(r)= & s_{n}+r s_{n-1}+\frac{r(r+1)}{2 !} s_{n-2} \\
& \quad \cdots+\frac{r(r+1) \cdots(r+n-1)}{n !} s_{0} \\
= & u_{n}+(r+1) u_{n-1}+\frac{(r+1)(r+2)}{2 !} u_{n-2} \\
& +\cdots+\frac{(r+1)(r+2) \cdots(r+n)}{n !} u_{0}, \\
&
\end{aligned}
$$

it is said that the series (1) has the sum $s$. We then say that the series (1) is summable $(C r)$ to the sum $s$. The least value of $r$ (assumed to be an integer) for which the limit in (5)

\footnotetext{
* Bulletin des Sciences mathématiques, ser. 2, vol. 14 (1890), pp. 114-120.
} 
exists is called the degree of indeterminacy of series (1), and the series is said to be $r$-fold indeterminate. Cesàro showed that if the series $u$ and $v$ are $p$-fold and $q$-fold indeterminate, respectively, then the product series $w$ is at most $(p+q+1)$ fold indeterminate.

It is easy to verify that the sum $S_{n}{ }^{(r)}$ employed above may be defined by the relations

$$
\begin{aligned}
& S_{n}{ }^{(1)}=s_{0}+s_{1}+\cdots+s_{n}, \\
& S_{n}{ }^{(2)}=S_{0}{ }^{(1)}+S_{1}{ }^{(1)}+\cdots+S_{n}^{(1)}, \\
& \cdot \cdot \cdot \cdot \cdot \cdot \cdot \cdot \cdot \cdot \cdot \cdot \cdot \cdot \cdot \\
& S_{n}{ }^{(r)}=S_{0}{ }^{(r-1)}+S_{1}^{(r-1)}+\cdots+S_{n}{ }^{(r-1)} .
\end{aligned}
$$

Also we have

$$
\lim _{n=\infty} \frac{(r+1)(r+2) \cdots(r+n)}{n !} \cdot \frac{r !}{n^{r}}=\lim _{n=\infty} \frac{(n+r) !}{n ! n^{r}}=1 .
$$

Hence in Cesàro's definition we may replace (5) by its equivalent

$$
s=\lim _{n=\infty} n^{-r} \cdot r ! \cdot S_{n}{ }^{(r)} .
$$

In 1880, ten years before the work of Cesàro, the limit in (5) for the case $r=1$ was considered by Frobenius* who showed that

$$
\lim _{x=1-0} \sum_{i=0}^{\infty} u_{i} x^{i}=\lim _{n=\infty} \frac{s_{0}+s_{1}+\cdots+s_{n}}{n+1}
$$

whenever the limit on the right exists and is finite. The limit in the first member of this relation was treated further by Hölder $\dagger$ in 1882 . In order to state his result conveniently let us write

$$
\begin{aligned}
& s_{n}{ }^{(0)}=s_{n}, \\
& s_{n}{ }^{(1)}=\frac{1}{n+1}\left(s_{0}{ }^{(0)}+s_{1}{ }^{(0)}+\cdots+s_{n}{ }^{(0)}\right),
\end{aligned}
$$

* Journal für Mathematik, vol. 89 (1880), pp. 262-264.

$\dagger$ Math. Annalen, vol. 20 (1882), pp. 535-549. This limit also appeared in the work of Euler and Abel. More recently it has been prominent in the literature of summable series. 


$$
\begin{aligned}
& s_{n}^{(2)}=\frac{1}{n+1}\left(s_{0}^{(1)}+s_{1}^{(1)}+\cdots+s_{n}{ }^{(1)}\right), \\
& \left.\cdot \cdot \cdot \cdot \cdot \cdot \cdot \cdot \cdot \cdot \cdot \cdot \cdot \cdot \cdot \cdot s_{n}^{(r-1)}\right) . \\
& s_{n}^{(r)}=\frac{1}{n+1}\left(s_{0}^{(r-1)}+s_{1}^{(r-1)}+\cdots+s^{(r)}\right.
\end{aligned}
$$

Hölder shows that

$$
\lim _{x=1-0} \sum_{i=0}^{\infty} u_{i} x^{i}=\lim _{n=\infty} s_{n}{ }^{(r)}
$$

provided that the last limit exists and is finite. If we denote the value of this limit by $s$ we may extend the notion of sum of an infinite series, defining the sum of (1) to be this number $s$. When $\lim _{n=\infty} s_{n}{ }^{(r)}$ exists and has the finite value $s$ we say that (1) is summable $(H r)$ to the sum $s$.

It will be observed that the Hölder and Cesàro definitions of sum of an infinite series (and the usual definition of sum of a convergent series as well) are special cases of the following more general definition:* The series (1) is said to have the sum $t$ in case $\lim _{n=\infty} t_{n}$ exists and has the finite value $t$, where

$$
t_{n}=c_{n 0} s_{0}+c_{n 1} s_{1}+\ldots+c_{n n} s_{n}, \quad c_{n n} \neq 0,
$$

the $c_{i j}$ being (real or complex) constants. Any method of summation belonging to the general class indicated by this definition will be called a method of mean values with finite reference.

It is obvious that a definition equivalent to the foregoing may be obtained by taking for $t_{n}$ the value $\dagger$

$$
t_{n}=a_{n 0} u_{0}+a_{n 1} u_{1}+\cdots+a_{n n} u_{n}, \quad a_{n n} \neq 0,
$$

* Among those who have treated this general type of definition may be mentioned Silverman, Dissertation (Missouri), 1910, [University of Missouri Studies, 1913]; Toeplitz, Prace matematyczno fizyczno, vol. 22 (1911), p. 113; Smail, Dissertation (Columbia), 1913; Schur, Math. Annalen, vol. 74 (1913), p. 447; Hurwitz and Silverman, Transactions Amer. Math. Society, vol. 18 (1917), p. 1; Kojima, Tôhoku Math. Journal, vol. 12 (1917), p. 291.

$\uparrow$ These definitions may be generalized by taking the coefficients $a_{i j}$ (and similarly the coefficients $c_{i j}$ ) to be functions of parameters $x_{1}, x_{2}$, $\ldots, x_{r}$. Then $t_{n}$ becomes $t_{n}\left(x_{1}, x_{2}, \ldots, x_{r}\right)$ and the sum of the series may be said to be the value of the limit

$$
\lim _{x_{1}=l_{1}} \lim _{x_{2}=l_{2}} \ldots \lim _{x_{r}=l_{r}} \lim _{n=\infty} t_{n}\left(x_{1}, x_{2}, \ldots, x_{r}\right)
$$

when this repeated limit exists and is finite. In this general form the definition has been treated by James (Columbia dissertation, 1917). See also the papers referred to at the end of this section. 
where the $a_{i j}$ are (real or complex) constants. In fact, the functions $t_{n}$ of $n$ in (6) and (7) are identical in case

$$
\begin{aligned}
a_{n n} & =c_{n n}, \\
a_{n, n-1} & =c_{n n}+c_{n, n-1}, \\
a_{n, n-2} & =c_{n n}+c_{n, n-1}+c_{n, n-2}, \\
\cdots & \cdots \cdots+\ldots \ldots+c_{n 0} .
\end{aligned}
$$

These relations are obviously equivalent to the following:

$$
\begin{aligned}
c_{n n} & =a_{n n}, \\
c_{n, n-1} & =a_{n, n-1}-\dot{a}_{n, n}, \\
c_{n, n-2} & =a_{n, n-2}-a_{n, n-1}, \\
\cdots \cdots & \cdots \cdots \\
c_{n 0} & =a_{n 0}-a_{n 1} .
\end{aligned}
$$

Certain other particular cases of summation by the method of mean values should be mentioned.

In 1907 Knopp* generalized Cesàro's definition of sum by taking for the sum $s$ of (1) the value

where

$$
s=\lim _{n=\infty} \frac{\Gamma(r+1)}{n^{r}} S_{n}^{(r)},
$$

$$
S_{n}{ }^{(r)}=\sum_{k=0}^{n} \frac{\Gamma(r+n-k+1)}{\Gamma(r+1) \Gamma(n-k+1)} u_{k},
$$

the symbol $\Gamma$ denoting the gamma function. For positive integral values of $r$ this is the same as Cesàro's definition.

De la Vallee Poussin† in 1908 defined the sum of (1) to be the number $s$ in case

$$
\lim _{n=\infty}\left(u_{0}+\sum_{k=1}^{n} \frac{n(n-1) \cdots(n-k+1)}{(n+1)(n+2) \cdots(n+k)} u_{k}\right)
$$

exists and has the finite value $s$.

In 1909 Ford $\ddagger$ gave a generalization of Cesàro's method of

* Sitzungsber. d. Berliner Math. Gesellschaft, Nov., 1907, pp. 1-12.

$\dagger$ Belg. Bulletin d. Sciences, 1908, pp. 193-254. See also a generalization by Kogbetlianz, Paris C. R., vol. 164 (1917), pp. 510-513, 626-628, 778780.

$\ddagger$ This Bulletin, vol. 15 (1909), pp. 439-444. 
summation in accordance with which the sum of (1) is said to have the value $s$,

$$
s=\lim _{n=\infty} \frac{f_{p}(n, r) s_{0}+f_{p}(n-1, r) s_{1}+\cdots+f_{p}(0, r) s_{n}}{f_{p}(n, r)+f_{p}(n-1, r)+\cdots+f_{p}(1, r)+f_{p}(0, r)},
$$

provided this limit exists, the function $f_{p}(n, r)$ being defined by the relation

$$
f_{p}(n, r)=\frac{r(r+1)}{r+\log _{p} n} \prod_{k=2}^{n} \frac{r+\log _{p} k}{\log _{p} k}
$$

where $\log _{0} n=n$ and $\log _{p} n=\log \left(\log _{p-1} n\right), p=1,2,3, \cdots$. For $p=0$ this reduces to Cesàro's definition.

Chapman* in 1911 developed the theory of Cesàro's mean value process when the order $r$ of summability is any real number other than a negative integer, employing for this purpose the Cesàro formulæ which we have given above (see equation (5) and those immediately following it) but without Cesàro's restriction that $r$ shall be integral. $\dagger$

Silverman (l. c., page 37 ) says that (1) is $\varphi$-summable to the sum $s$ in case

$$
\lim _{n=\infty} \frac{\varphi_{0} s_{0}+\varphi_{1} s_{1}+\cdots+\varphi_{n} s_{n}}{n+1}=s, \quad \lim _{n=\infty} \varphi_{n}=1 .
$$

On account of needs arising in the study of Dirichlet series M. Riesz $\ddagger$ was led to define the sum of (1) as the finite number $s$ in case

$$
\lim _{\omega=\infty} \omega^{-r} \sum_{\lambda_{k}<\omega}\left(\omega-\lambda_{k}\right)^{r} u_{k}=s,
$$

where $\lambda_{0} \geqq 0, \lambda_{1}, \lambda_{2}, \ldots$ is a sequence of distinct real numbers tending monotonically to infinity with $n$ and the sum for each $\omega$ is taken for all $\lambda_{k}<\omega$. When the limit in (12) exists and is finite we shall say (with Hardy§) that (1) is summable $(R \lambda r)$, that is, summable by Riesz's means of type $\lambda$ and order $r$.

It will be observed that this definition differs from the pre-

* Proc. London Math. Society, ser. 2, vol. 9 (1911), pp. 369-409.

$\dagger$ An extension of Cesàro's definition to double series has been given by C. N. Moore, Transactions Amer. Math. Society, vol. 14 (1913), pp. 73-104.

† Paris C. R., vol. 152 (1911), pp. 1651-1654. See also an earlier note by Riesz in Paris $C$. $R$., vol. 149 (1909), 18-20. This paper contains an error which is corrected in the later communication.

§ Proc. London Math. Society, ser. 2, vol. 8 (1909), pp. 301-320. 
ceding ones in that the limit is taken with respect to the continuous variable $\omega$ rather than with reference to a variable $n$ running over the set of non-negative integers. Following out the suggestion thus arising, one might modify the definition associated with (6) and (7) so that the limit with respect to $n$ shall be replaced by a limit with respect to a continuous variable. Compare the discussion associated with (13).

Several considerations have led investigators to introduce other definitions of sum than those associated with the method of mean values with finite reference. In his now classic researches Borel was influenced by the fact that the method of Cesàro suffices to sum only a relatively restricted class of series (see $\$ 5$ below). Accordingly he introduced* the method of mean values with infinite reference. In a form convenient for our purposes the method may be stated thus: $\dagger$ The series (1) is said to have the sum $t$ in case $\lim _{n=\infty} t_{n}$ exists and has the finite value $t$, where

$$
t_{n}=\sum_{k=0}^{\infty} c_{n k} s_{k},
$$

the $c_{i j}$ being (real or complex) constants.

For an alternative definition one may define $t_{n}$ by the relation

$$
t_{n}=\sum_{k=0}^{\infty} a_{n k} u_{k}
$$

where the $a_{i j}$ are (real or complex) constants.

In order to specialize the definition associated with (13) into a conveniently workable form one may take for $c_{n k}$ the value $c_{k} n^{k} / \varphi(n)$, where

$$
\varphi(a)=c_{0}+c_{1} a+c_{2} a^{2}+c_{3} a^{3}+\cdots,
$$

and modify the limit operation by replacing $n$ by the continuous variable $a$ so as to have for the sum of (1) the (finite) value $t$, where

$$
t=\lim _{a=\infty} \frac{c_{0} s_{0}+c_{1} a s_{1}+c_{2} a^{2} s_{2}+\cdots}{\varphi(a)} .
$$

* Leçons sur les Séries divergentes, 1901, pp. 91-98. Here will be found references to Borel's earlier work on this subject.

$\dagger$ Here the variable $n$ may run over the range of integers or over a continuous range. 
For detailed study Borel chooses* for $\varphi(a)$ the function $e^{a}$, so that he defines the sum $t$ of (1) by the more special relation

$$
t=\lim _{a=\infty} e^{-a}\left(s_{0}+s_{1} \frac{a}{1}+s_{2} \frac{a^{2}}{2 !}+s_{3} \frac{a^{3}}{3 !}+\cdots\right) .
$$

This definition of sum gives rise to what Borel calls the exponential method of summation of series. It is obviously a special case of the method of mean values with infinite reference.

For the sum $t$ of (1) LeRoy $\dagger$ has taken the value

$$
t=\lim _{x=1-0} \sum_{k=0}^{\infty} \frac{\Gamma(k x+1)}{\Gamma(k+1)} u_{k},
$$

provided that this limit exists and is finite. He was led to this definition through a consideration of the problem of analytic continuation of a function defined by a power series.

It is obvious that one may extend the definition in (13) by considering the coefficients $c_{i j}$ or $a_{i j}$ to be functions of parameters $x_{1}, x_{2}, \ldots, x_{r}$ and taking the sum of (1) to be

$$
\lim _{x_{1}=l_{1}} \lim _{x_{2}=l_{2}} \cdots \lim _{x_{r}=l_{r}} \lim _{n=\infty} t_{n}
$$

when this repeated limit exists and is finite. For a treatment of some such definitions see the papers referred to at the end of this section, especially those of Hardy and Chapman, Chapman, and Smail.

An easy and natural stepł leads one from Borel's exponential method of summation to his integral method. Denoting by $s(a)$ the function

$$
s(a)=s_{0}+s_{1} \frac{a}{1}+s_{2} \frac{a^{2}}{2 !}+s_{3} \frac{a^{3}}{3 !}+\cdots,
$$

one has from (14) the relation

$$
t-u_{0}=\left[e^{-a} s(a)\right]_{0}^{\infty}=\int_{0}^{\infty} \frac{d}{d a}\left[e^{-a} s(a)\right] d a,
$$

\footnotetext{
* Interesting applications are also made of certain other cases. See the papers referred to in the second preceding footnote. In particular, considerable treatment (Séries divergentes, pp. $129 \mathrm{ff}$.) is given of the case in which $\varphi(a)=e^{a^{k}}$.

$\dagger$ Annales Fac. Sci. Toulouse, ser. 2, vol. 2 (1900), pp. 317-430; see especially pp. $327-328$.

$\ddagger$ Borel, 1. c., pp. 97-100. On Borel's generalizations of the notion of limit see also Hanni, Monatshefte für Mathematik und Physik, vol. 12 (1901), pp. 265-289.
} 
provided that

But

$$
\lim _{a=0} e^{-a} s(a)=u_{0}
$$

$$
\begin{aligned}
\frac{d}{d a}\left[e^{-a} s(a)\right] & =e^{-a}\left[s^{\prime}(a)-s(a)\right] \\
& =\left(s_{1}-s_{0}\right)+\frac{\left(s_{2}-s_{1}\right) a}{1 !}+\frac{\left(s_{3}-s_{2}\right) a^{2}}{2 !}+\cdots \\
& =u_{1}+u_{2} \frac{a}{1 !}+u_{3} \frac{a^{2}}{2 !}+\cdots
\end{aligned}
$$

Denoting by $\bar{u}(a)$ the function in the last member and integrating by parts, we have

$$
\begin{aligned}
t-u_{0}=\int_{0}^{\infty} & e^{-a} \bar{u}(a) d a \\
& =\left[e^{-a} \int_{0}^{a} \bar{u}(a) d a\right]_{0}^{\infty}+\int_{0}^{\infty} e^{-a}\left[\int_{0}^{a} \bar{u}(a) d a\right] d a ;
\end{aligned}
$$

whence it follows that

$$
t=\int_{0}^{\infty} e^{-a} u(a) d a
$$

where

$$
u(a)=u_{0}+\frac{u_{1} a}{1 !}+\frac{u_{2} a^{2}}{2 !}+\frac{u_{3} a^{3}}{3 !}+\cdots .
$$

In the detailed development of the theory it is assumed by Borel that the series (1) is such that the associated function $u(a)$ is an entire function. Then when the integral in (16) exists he takes its value $t$ to be the sum of (1) and says that the series is summable to the sum $t$.

Furthermore, if the integrals

$$
\int_{0}^{\infty} e^{-a}|u(a)| d a, \quad \int_{0}^{\infty}\left|e^{-a}\right| u^{(\lambda)} a \mid d a \quad(\lambda=1,2,3, \cdots),
$$

exist, where $\lambda$ is an index of differentiation, then Borel says that (1) is absolutely summable.

Borel has also employed a generalization of his definition (16) in which

$$
t=\int_{0}^{\infty} e^{-a} u_{p}(a) d a
$$


where

$$
\begin{aligned}
u_{p}(a)=\left(u_{0}+u_{1}+\right. & \left.\cdots+u_{p-1}\right) \\
& +\left(u_{p}+u_{p+1}+\cdots+u_{2 p-1}\right) \frac{a}{1 !} \\
& +\left(u_{2 p}+u_{2 p+1}+\cdots+u_{3 p-1}\right) \frac{a^{2}}{2 !}+\cdots
\end{aligned}
$$

For a discussion of the definitions of LeRoy see $\$ 7$ below.

It is convenient to insert here also references to the methods of Euler,* Buhl, $\dagger$ Stieltjes, $\ddagger$ Hardy and Chapman, $\S$ Chapman,\| Barnes, $\mathbb{T}$ Smail,** James, †† C. N. Moore, W. H. Young, |||| and Cunningham. $\uparrow \uparrow$

For the sake of unity and economy of space we shall not treat the question of uniform summability, particularly since the notion of uniformity enters in essentially an obvious way.

\section{\$2. Regularity of a Definition of Sum by the Method of Mean Values with Finite Reference.}

A definition of sum of an infinite series is said to be regular*** if it assigns to every convergent series the same sum as the usual definition. It is desirable (and natural) to confine attention to those definitions which are regular in this sense.

We shall now determine necessary and sufficient conditions that the general definition of sum by the method of mean

* Euler's treatment of divergent series (Inst. Calc. Diff., Pars II, Cap. I) depends on a transformation resulting in convergent series when applied to certain classes of divergent series. See the treatment of this method by Bromwich, Infinite Series, pp. 302-310.

† Bulletin des Sciences mathématiques, vol. 42 (1907), pp. 340-346; Journal de Mathématiques, ser. 6, vol. 4 (1908), pp. 367-377.

$\ddagger$ Annales de Toulouse, 8J, pp. 1-122; 9A, pp. 1-47; 1894-1895.

\$ Quarterly Journal of Mathematics, vol. 42 (1911), pp. 181-215. A general class of definitions forms the subject matter of this paper. A brief statement of the guiding principle is given in $\$ 8$ below.

|| Quarterly Journal of Mathematics, vol. 43 (1911), pp. 1-52.

T Phil. Transactions Royal Society, 199A (1902), pp. 411-500.

** Columbia dissertation, 1913.

†† Columbia dissertation, 1917.

\$ Transactions Amer. Math. Society, vol. 8 (1907), pp. 299-330; vol. 14 (1913), pp. 73-104.

\$\& Annales de Toulouse, ser. 2, vol. 1 (1899), pp. 117-175.

II Leipaiger Berichte, vol. 63 (1911), pp. 369-387.

ๆ Proc. London Math. Society, ser. 2, vol. 3 (1905), pp. 157-169.

$* * *$ The term "regular" is used in essentially the sense of the text by Hurwitz and Silverman, Transactions Amer. Math. Society, vol. 18 (1917), pp. $1-0$. 
values with finite reference shall be regular. Employing the notation of equation (6) and using the definition of regularity, we see that we must have $\lim _{n=\infty} t_{n}=\lim _{n=\infty} s_{n}$ for every convergent sequence $s_{0}, s_{1}, s_{2}, \ldots$. Then if we take $s_{k}=1$ and $s_{n}=0$ when $n \neq k$, we have $\lim _{n=\infty} c_{n k}=0$ for every $k$. Again, taking $s_{0}=1=s_{1}=s_{2}=\cdots$, we find the second of the following two necessary conditions:

$$
\lim _{n=\infty} c_{n k}=0, \quad \lim _{n=\infty}\left(c_{n 0}+c_{n 1}+\cdots+c_{n n}\right)=1 .
$$

In what follows we employ these conditions.

Consider the superior limit

$$
\limsup _{n=\infty} A_{n} \text {, where } A_{n}=\left|c_{n 0}\right|+\left|c_{n 1}\right|+\cdots+\left|c_{n n}\right| \text {. }
$$

In view of (17) its value must be either infinity or a positive number not less than unity. Suppose first that its value is infinity. We shall determine a sequence $s_{0}, s_{1}, s_{2}, \ldots$ approaching zero such that $t_{n}$ does not approach a finite limit as $n$ approaches infinity. Let $\alpha$ be a number greater than 1 and choose $n$, so that $A_{n_{1}}>\alpha^{2}$. Then put*

$$
s_{i}=\frac{1}{\alpha} \frac{\left|c_{n_{1} i}\right|}{c_{n_{1} i}} \quad\left(i=0,1, \cdots, n_{1}\right) .
$$

Then $\left|t_{n_{1}}\right|>\alpha$. Choose $n_{2}$, greater than $n_{1}$, so that

$$
\left|c_{n_{2}}\right|+\cdots+\left|c_{n_{2} n_{1}}\right|<\alpha, \quad A_{n_{2}}>\alpha^{4}+2 \alpha^{2} .
$$

Then put

$$
s_{i}=\frac{1}{\alpha^{2}} \frac{\left|c_{n_{2} i}\right|}{c_{n_{2} i}} \quad\left(i=n_{1}+1, \cdots, n_{2}\right) .
$$

It is easy to see that $\left|t_{n_{2}}\right|>\alpha^{2}$; for

$$
\begin{aligned}
\left|t_{n_{2}}\right| & \geqq\left|\sum_{i=n_{1}+1}^{n_{2}} c_{n_{2} i} s_{i}\right|-\sum_{i=0}^{n_{1}}\left|c_{n_{2} i} s_{i}\right| \\
& \geqq\left|\frac{1}{\alpha^{2}} A_{n_{2}}-\frac{1}{\alpha^{2}} \sum_{i=0}^{n_{1}}\right| c_{n_{2} i}||-\sum_{i=0}^{n_{1}}\left|c_{n_{2} i} s_{i}\right|>\alpha^{2} .
\end{aligned}
$$

Similarly, choose $n_{3}$ greater than $n_{2}$ and such that

$$
\left|c_{n_{3} 0}\right|+\cdots+\left|c_{n_{8} n_{2}}\right|<\alpha, \quad A_{n_{3}}>\alpha^{6}+3 \alpha^{3}
$$

* Whenever $c_{n_{i}}=0$ we understand that $\left|c_{n_{i}}\right| / c_{n_{i}}$ is to be replaced by unity here and in similar places below. 
and put

$$
s_{i}=\frac{1}{\alpha^{3}} \frac{\left|c_{n_{8} i}\right|}{c_{n_{8} i}} \quad\left(i=n_{2}+1, \cdots, n_{3}\right) .
$$

Then it may be shown that $\left|t_{n_{3}}\right|>\alpha^{3}$. Proceeding in this way we obtain a sequence $s_{0}, s_{1}, s_{2}, \cdots$, converging to zero, such that lim $\sup _{n=\infty}\left|t_{n}\right|=\infty$. Hence a third necessary condition for the regularity of our definition is that $\lim \sup _{n=\infty} A_{n}$ shall be finite.

We have thus established the necessity of the conditions named in the following theorem:*

Theorem I. A necessary and sufficient condition that a sum shall be assigned to every convergent series (1) by the definition associated with (6) and that its value shall be the usual sum $s$ of (1) is that

1) $\lim c_{n k}=0$ for every $k$;

2) $\lim _{n=\infty}\left(c_{n 0}+c_{n 1}+\cdots+c_{n n}\right)=1$;

3) a number $M$, independent of $n$, shall exist such that for every $n$

$$
\left|c_{n 0}\right|+\left|c_{n 1}\right|+\cdots+\left|c_{n n}\right|<M .
$$

It remains to prove the sufficiency of this condition. In view of 2), definition (6) and the fact that $\lim _{n=\infty}\left(s_{n}-s\right)$ is zero when $\lim _{n=\infty} s_{n}=s$ it is clearly sufficient to prove that $\lim _{n=\infty} t_{n}$ exists and is zero when $\lim _{n=\infty} s_{n}=0$. Employing the last relation we see that for every positive $\epsilon$ there exists an $N$ such that $\left|s_{n}\right|<\epsilon$ whenever $n>N$. But for $n>N$ we may write

$$
t_{n}=\left(c_{n 0} s_{0}+\cdots+c_{n N} s_{N}\right)+\sum_{i=N+1}^{n} c_{n i} s_{\imath} .
$$

From 1) it follows that the quantity in parenthesis here approaches zero as $n$ approaches infinity. In view of 3 ) the last sum is seen to be less than $M \epsilon$ in absolute value. Hence $\lim _{n=\infty} t_{n}=0$. This completes the proof of the theorem.

In the more usual definitions of summability by the method of mean values with finite reference the coefficients $c_{i j}$ are

* The sufficiency of this condition has been proved by Silverman (l. c.). The necessity of the condition has been proved by Toeplitz (l. c.). The theorem has been generalized by Kojima (l. c.), who also extends the corollary. Less complete results of like import have been given by several writers. See, among others, the papers referred to near the end of $\$ 1$. 
non-negative real constants. For this case it should be observed that condition 3 ) is implied by condition 2 ).

An obvious modification of the proof of Theorem I leads to the following corollary:

Corollary. A necessary and sufficient condition that $\lim _{n=\infty}\left(c_{n 0} s_{0}+c_{n 1} s_{1}+\cdots+c_{n n} s_{n}\right)$ shall exist and be finite in all cases in which $\lim _{n=\infty} s_{n}$ exists and is finite is that

1) $\lim _{n=\infty} c_{n k}$ shall exist and be finite for every $k$;

2) $\lim _{n=\infty}\left(c_{n 0}+c_{n 1}+\cdots+c_{n n}\right)$ shall exist and be finite;

3) a quantity $M$, independent of $n$, shall exist such that

$$
\left|c_{n 0}\right|+\left|c_{n 1}\right|+\cdots+\left|c_{n n}\right|<M \text { for every } n \text {. }
$$

In view of relations $\left(8^{\prime}\right)$ it is easy to show that the foregoing theorem may be put into the following equivalent form:

THEOREM II. A necessary and sufficient condition that a sum shall be assigned to every converging series (1) by the definition associated with (7) and that its value shall be the usual sum $s$ of (1) is that

1) $\lim a_{n k}=1$ for every $k$;

2) a constant $M$, independent of $n$, shall exist such that for every $n$

$$
\sum_{i=0}^{n-1}\left|a_{n, i}-a_{n, i+1}\right|<M
$$

In case $a_{n k}$ is positive and $a_{n k}-a_{n, k+1}$ is positive [negative] for every $n$ and $k$ it is clear that condition 2 ) is a consequence of condition 1 ).

It should be observed that the demonstration of Theorems I and II and the corollary to I requires no use of the hypothesis $c_{n n} \neq 0, a_{n n} \neq 0$.

It is an immediate consequence of Theorems I and II that Cesàro's definitions of sum are regular; and likewise that Knopp's and Chapman's extensions of them are regular. It is not difficult to establish similarly the regularity of Ford's extensions of Cesàro's definitions. In order to prove that Hölder's definition of order $r$ is regular we observe that it is an immediate consequence of Theorem I that $\lim _{n=\infty} s_{n}{ }^{(r)}=s$ whenever $\lim _{n=\infty} s_{n}{ }^{(r-1)}=s$, and hence whenever $\lim _{n=\infty} s_{n}{ }^{(r-2)}$ $=s, \cdots$, and hence finally whenever $\lim _{n=\infty} s_{n}{ }^{(0)}=s$. From Theorem II it follows at once that de la Vallée Poussin's definition is regular. The regularity of other definitions by the 
method of mean values with finite reference may be treated readily by the aid of Theorems I and II.

\section{§3. Mutual Consistency of Two Definitions of Sum by the Method of Mean Values with Finite Reference.}

We shall say that two definitions of sum of an infinite series are mutually consistent* whenever it is true that the same sum is assigned by them to every series that is summable in accordance with both definitions.

Either one of the limits (see Hurwitz and Silverman, 1. c., page 1)

$$
\lim _{n=\infty} \frac{1}{n} \sum_{k=1}^{n} s_{k}, \quad \lim _{n=\infty} \frac{1}{n} \sum_{k=2}^{n}\left[1+\frac{(-1)^{k+1}}{\log k}\right] s_{k}
$$

affords a regular definition of the sum of a series (1). But the two definitions are not mutually consistent, since the first limit has the value 0 and the second the value 1 if $s_{n}=(-1)^{n+1} \log n$.

Let us consider the problem as to the mutual consistency of the definition associated with relation (6) and the following in which (1) is said to have the sum $T=\lim _{n=\infty} T_{n}$, where

$$
\begin{array}{rlrl}
T_{n} & =r_{n 0} s_{0}+r_{n 1} s_{1}+\ldots+r_{n n} s_{n}, & & r_{n n} \neq 0, \\
& =\alpha_{n 0} u_{0}+\alpha_{n 1} u_{1}+\ldots+\alpha_{n n} u_{n}, & \alpha_{n n} \neq 0 .
\end{array}
$$

This fundamental problem relative to definitions by the method of mean values with finite reference seems not to have been resolved. In fact, so far as I am aware, it has received attention in a general way only in a single investigation, namely in the paper by Hurwitz and Silverman (already referred to), where the mutual consistency of all definitions of a certain subclass of these definitions has been established. We shall not reproduce the results of these authors.

Among the special cases of mutual consistency there is one of great importance which we shall treat further, namely, that in which every series which is summable to the sum $s$ by a given one of the two given methods is also summable to the same sum $s$ by the other of the two methods. In the preceding section we saw that Hölder's definitions are mutually

\footnotetext{
* Hurwitz and Silverman (1. c.) have used the term "consistent" in the sense of our term "mutually consistent."
} 
consistent, the sum $(H r)$ existing and agreeing with the sum $(H k)$ for $k<r$ whenever the latter sum exists.

Let us consider the definitions of sum associated with relations (6) and (18) respectively. It is convenient to employ the infinite matrices

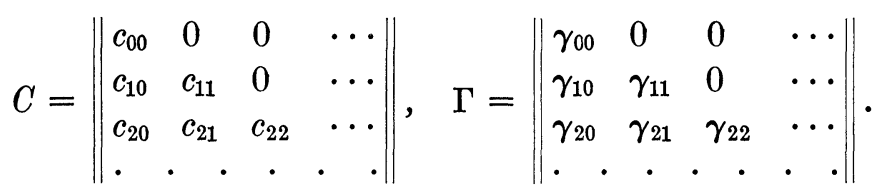

Whenever conditions 1), 2), 3) of theorem I are satisfied we shall say that the matrix $C$ is a regular matrix of the first kind; and likewise of course for other matrices of the same form.

In matrix notation we may write relations (6) and (18) in the respective forms

$$
\left(t_{n}\right)=C\left(s_{n}\right), \quad\left(T_{n}\right)=\Gamma\left(s_{n}\right) .
$$

If we solve the first of these relations for the $s$ 's, obtaining $\left(s_{n}\right)=C^{-1}\left(t_{n}\right)$, and substitute into the second, we have

$$
\left(T_{n}\right)=\Gamma C^{-1}\left(t_{n}\right)
$$

where $\Gamma C^{-1}$ denotes the product of $\Gamma$ and $C^{-1}$. Employing theorem I we have the following results (already essentially contained in Theorem I itself):

TheOREM III. A necessary and sufficient condition that the definition associated with (18) shall assign a sum $t$ to every series (1) to which the definition associated with (6) assigns the sum $t$ is that the matrix $\Gamma C^{-1}$ shall be a regular matrix of the first kind.

This theorem may also be stated simply in terms of the matrices

$$
A=\left\|\begin{array}{llll}
a_{00} & 0 & 0 & \cdots \\
a_{10} & a_{11} & 0 & \cdots \\
a_{20} & a_{21} & a_{22} & \cdots \\
. & . & . & .
\end{array}\right\|, \quad \alpha=\left\|\begin{array}{llll}
\alpha_{00} & 0 & 0 & \cdots \\
\alpha_{10} & \alpha_{11} & 0 & \cdots \\
\alpha_{20} & \alpha_{21} & \alpha_{22} & \cdots \\
. & . & . & .
\end{array}\right\| .
$$

We shall say that a matrix $A$ is a regular matrix of the second kind whenever conditions 1) and 2) of Theorem II are satisfied.

We may write (7) in the form $\left(t_{n}\right)=A\left(s_{n}\right)$, whence we have $\left(s_{n}\right)=A^{-1}\left(t_{n}\right)$. In view of Theorem II we may now state the following result (already essentially contained in Theorem II itself): 
Theorem IV. A necessary and sufficient condition that the definition associated with (18) shall assign a sum t to every series (1) to which the definition associated with (7) assigns the sum $t$ is that the matrix $\alpha A^{-1}$ shall be a regular matrix of the second kind.

It may be shown that Knopp (and hence Cesàro) summability of order $r_{1}$ assigns the sum $s$ to any series to which this sum is assigned by the same summability of order $r_{2}$ where $r_{1}>r_{2}>-1$ (Knopp, l. c., page 5). A similar result holds also in the case of Chapman summability (Chapman, l. c.).

In the Paris Comptes Rendus for June, 1914, T. H. Gronwall and C. N. Moore independently proved that every series which is summable $(C r)$ to the sum $s$ is summable to the same sum by the method of de la Vallée Poussin; and that there are series summable by the latter method but not by the method of Cesàro for any value of the order $r$.

\section{\$4. Equivalence of Two Definitions of Sum by the Method of Mean Values with Finite Reference.}

We shall say that two definitions of sum of an infinite series are equivalent whenever it is true that every series which has a sum $s$ in accordance with either of these definitions also has the same sum $s$ in accordance with the other definition.

As a corollary from Theorems III and IV of the preceding section we now have immediately the following theorem:

Theorem V. A necessary and sufficient condition that the definitions of sum of an infinite series associated with (6) [or (7)] and (18), respectively, shall be equivalent may be put in either of the following two forms:

1) The matrices $\Gamma C^{-1}$ and $C \Gamma^{-1}$ shall both be regular of the first kind;

2) The matrices $\alpha A^{-1}$ and $A \alpha^{-1}$ shall both be regular of the second kind.

By means of this theorem Schur* has given a demonstration of the equivalence of summability $(\mathrm{Cr} r$ ) and summability $(\mathrm{Hr})$. That Hölder summability implies that of Cesàro was first proved by Knopp. $\dagger$ Independently, and by different methods, Schnee $\ddagger$ and Ford§ showed that Cesàro summability implies

* Math. Annalen, vol. 74 (1913), pp. 447-458.

+ Dissertation, Berlin, 1907.

$\ddagger$ Math. Annalen, vol. 67 (1909), pp. 110-125.

$\S$ Amer. Journal of Mathematics, vol. 32 (1910), pp. 315-326. 
that of Hölder. More recently Faber* and Watanabe† have given other demonstrations of the equivalence of summability $(\mathrm{Cr})$ and summability $(\mathrm{Hr}) . \ddagger$

Silverman (Dissertation, page 37) has established the equivalence of summability $(C 1)$ and his $\varphi$-summability provided that $\varphi_{n}$ approaches 1 monotonically as $n$ increases indefinitely. He has pointed out (l. c., page 44) how this affords a convenient test for summability $(C 1)$, and has indicated a means of obtaining an (unsatisfactory) extension of the method to the case of summability $(C r)$.

\section{§5. Further Consideration of Definitions by the Method of Mean Values with Finite Reference.}

Besides the condition of being regular, already imposed upon our definitions of sum of divergent series, it is evidently desirable to restrict these definitions so that, as far as possible, the usual rules for operating with convergent series shall also be valid for summable divergent series.

Probably the first additional requirement to be demanded in the case of a given definition is that either of the series

$$
\begin{gathered}
u_{0}+u_{1}+u_{2}+\cdots, \\
u_{1}+u_{2}+\cdots,
\end{gathered}
$$

shall have a sum whenever the other has and that $t_{0}$ shall be equal to $t-u_{0}$ if $t$ and $t_{0}$ are their respective sums.

Let us consider the general definition associated with (6) subject to the conditions of regularity as given in Theorem I. Let us write

$$
\begin{aligned}
& t_{n}=c_{n 0} s_{0}+c_{n 1} s_{1}+\cdots+c_{n n} s_{n}, \\
& \bar{t}_{n}=c_{n 0} s_{1}+c_{n 1} s_{2}+\cdots+c_{n n} s_{n+1} .
\end{aligned}
$$

Then if (19) has the sum $t$ we have $\lim _{n=\infty} t_{n}=t$, while if (20) has the sum $t-u_{0}$ we have $\lim _{n=\infty} \bar{t}_{n}=t$. Hence, a necessary and sufficient condition that our requirement shall be met is that either of the quantities $t_{n}, \bar{t}_{n}$ shall approach a (finite)

* Münchener Sitzungsberichte, 1913, pp. 519-531.

† Tôhoku Mathematical Journal, vol. 5 (1914), pp. 21-28.

¥ In this connection see also Landau's paper on the corresponding problem for integrals, Leipziger Berichte, vol. 65 (1913), pp. 131-138, and Fekete's paper on "absolute summability" by the methods of Hölder and Cesàro, Math. és térmesz., vol. 32 (1914), pp. 389-425. 
limit $t$ whenever the other does; and hence that either of the quantities $t_{n}{ }^{\prime}, \bar{t}_{n}$ shall approach a finite limit $t$ whenever the other does, where

$$
t_{n}{ }^{\prime}=c_{n+1,1} s_{1}+\cdots+c_{n+1, n+1} s_{n+1},
$$

since $\lim _{n=\infty} c_{n 0}=0$.

Symbolically, we may write $\left(t_{n}{ }^{\prime}\right)=D\left(s_{n+1}\right)$, where $D$ denotes the infinite matrix

$$
D=\left\|\begin{array}{llll}
c_{11} & 0 & 0 & \cdots \\
c_{21} & c_{22} & 0 & \cdots \\
c_{31} & c_{32} & c_{33} & \cdots \\
\cdot & \cdot & \cdot & \cdot
\end{array}\right\| .
$$

Similarly, we may write $\left(t_{n}\right)=C\left(s_{n+1}\right)$, where $C$ denotes the matrix represented by this symbol in $\$ 3$.

From considerations precisely like those involved in proving Theorem I we are now led to the following result:

Theorem VI. A necessary and sufficient condition that a regular definition of the form associated with (6) shall assign a sum to either of the series $u_{0}+u_{1}+u_{2}+\cdots$ and $u_{1}+u_{2}$ $+\cdots$ whenever it assigns a sum to the other and that $t_{0}$ shall be equal to $t-u_{0}$ where $t$ and $t_{0}$ are respectively the sums of these series, is that each of the matrices $D C^{-1}$ and $C D^{-1}$ shall be regular of the first kind.

A second natural requirement is the following: If a definition assigns sums $t^{\prime}$ and $t^{\prime \prime}$ to the first two of the series

$$
\sum_{n=0}^{\infty} u_{n}, \quad \sum_{n=0}^{\infty} v_{n}, \quad \sum_{n=0}^{\infty}\left(u_{n} \pm v_{n}\right)
$$

it shall assign the sum $t^{\prime} \pm t^{\prime \prime}$ to the last of these series. This requirement is obviously met by all definitions by the method of mean values with finite reference.

Again, it is clear that such a definition assigns the sum $k t$ to the series $k u_{0}+k u_{1}+\cdots$ whenever it assigns the sum $t$ to the series $u_{0}+u_{1}+\cdots$.

So far as definitions by the method of mean values with finite reference are concerned we need to ascertain when the first alone of these additional requirements is met. For this we may utilize Theorem VI or we may proceed directly in the case of a particular definition. Thus it may be shown in 
particular that this requirement is met by the definitions of Cesàro and Hölder.

Owing to the frequent use which has been made of Cesàro's definitions of sum it is desirable to refer to certain additional results associated with them.

Theorem VII. Necessary conditions in order that series (1) shall be summable $(C r)$ are that*

$$
\lim _{n=\infty} n^{-r} S_{n}^{(r-t)}=0, \quad 0<t \leqq r ; \quad \lim _{n=\infty} n^{-r} u_{n}=0 .
$$

From the definition of $S_{n}^{(r)}$ in $\S 1$ we have

$$
S_{n}{ }^{(r-1)}=S_{n}{ }^{(r)}-S_{n-1}{ }^{(r)} \text {. }
$$

But $n^{-r} S_{n}(r)$ approaches a finite limit $l$ as $n$ approaches infinity, since (1) is now summable $(C r)$. Hence

$$
\begin{aligned}
\lim _{n=\infty} n^{-r} S_{n}{ }^{(r-1)} & =\lim _{n=\infty}\left[n^{-r} S_{n}{ }^{(r)}-\left(\frac{n}{n-1}\right)^{-r}(n-1)^{-r} S_{n-1}(r)\right] \\
& =l-l=0 .
\end{aligned}
$$

If we now assume that $\lim _{n=\infty} n^{-r} S_{n}{ }^{(r-t)}=0$ for a given $t$ we have

$$
\begin{aligned}
& \lim _{n=\infty} n^{-r} S_{n}^{(r-t-1)} \\
& \quad=\lim _{n=\infty}\left[n^{-r} S_{n}^{(r-t)}-\left(\frac{n}{n-1}\right)^{-r}(n-1)^{-r} S_{n-1}{ }^{(r-t)}\right]=0 .
\end{aligned}
$$

Induction now yields the first set of conditions. The last is similarly proved by means of the relations $s_{n}-s_{n-1}=u_{n}$ and $\lim _{n=\infty} n^{-r} s_{n}=0$.

From this theorem it follows that Cesàro's method is inapplicable if, to put it roughly, $\left|u_{n}\right|$ is too large when $n$ is large. Hardy $\dagger$ has shown that the method is also inapplicable if $\left|u_{n}\right|$ is too small when $n$ is large and in such wise that series (1) is not convergent. One of his theorems is as follows:

Theorem VIII. If $n\left|u_{n}\right|<K$, a quantity independent of $n$, then series (1) is not summable $(\mathrm{Cr} r$ for any value of $r$ unless it is convergent.

Reference may be made to certain additional results which

* Bromwich, Infinite Series, p. 318, obtains the last result in a different way.

† Proc. London Math. Society, ser. 2, vol. 8 (1909), pp. 301-320. 
throw light upon the nature and the limitations of the methods of Cesàro, namely, those due to Fejer,* Hardy, † Chapman, $\ddagger$ Landau, \$ Kojima, \| Bohr, \ Ottolenghi, ** Hardy and Littlewood, $\dagger$ Bromwich,

\section{§6. Definitions of Sum by the Method of Mean Values with Infinite Reference.}

Let us now consider those definitions in which series (1) is said to have the sum $t$ in case $\lim _{r=\infty} t_{r}$ exists \|\| and has the finite value $t$, where

$$
t_{r}=\sum_{k=0}^{\infty} c_{r k} s_{k},
$$

the $c_{r j}$ being (real or complex) constants.

Again, we demand first of all that the definition shall be regular. In particular, we must have $\lim _{r=\infty} t_{r}=0$ when $s_{k}=1$ and $s_{i}=0$ for $i \neq k$. Hence $\lim _{r=\infty} c_{r k}=0$ for every $k$. Taking $s_{k}=1$ for every $k$ we see that for every permissible $r$ the series $c_{r 0}+c_{r 1}+c_{r 2}+\cdots$ must converge and that

$$
\lim _{r=\infty} \sum_{k=0}^{\infty} c_{r k}=1 \text {. }
$$

In order that $t_{r}$ shall be defined for every convergent series and for each permissible value of $r$ it is necessary (and sufficient) that $\lim _{n=\infty}\left(c_{r 0} s_{0}+c_{r 1} s_{1}+\cdots+c_{r n} s_{n}\right)$ shall exist and be finite in all cases in which $\lim _{n=\infty} s_{n}$ exists and is finite. Thence, in view of condition 3 ) in the corollary to Theorem I, we see that the series $\left|c_{r 0}\right|+\left|c_{r 1}\right|+\cdots$ must converge for every $r$.

* Math. Annalen, vol. 58 (1903), pp. 62-66.

$\dagger$ Proc. London Math. Society, ser. 2, vol. 6 (1908), pp. 255-264; ser. 2, vol. 8 (1909), pp. 301-320; Math. Annalen, vol. 64 (1907), pp. 77-94.

¥ Proc. London Math. Society, ser. 2, vol. 9 (1910), pp. 369-409.

Prace matematyczno-fizyczno, vol. 21 (1910), pp. 97-177.

Tôhoku Mathematical Journal, vol. 12 (1917), pp. 304-321.

T Paris C. R., vol. 148 (1909), pp. 75-80; Göttinger Nachrichten, 1909, pp. 247-262.

** Giornale di Matematiche, vol. 49 (1911), pp. 233-279.

†† Proc. London Math. Society, ser. 2, vol. 11 (1912), pp. 1-16; Palermo Rendiconti, vol. 41 (1916), pp. 36-53.

¥ Math. Annalen, vol. 65 (1908), pp. 313-349.

\$ Atti R. Accad. Sc. Torino, vol. 50 (1915), pp. 97-112.

II $\|$ The limiting variable $r$ may approach infinity over the set of numbers $1,2,3, \ldots$ or over the continuum of real numbers. The treatment applies to both cases simultaneously. 
If, in addition, a quantity $M$, independent of $r$, exists such that

$$
\sum_{k=0}^{\infty}\left|c_{r k}\right|<M \text { for every } r,
$$

it is easy to show that the definition is regular. For suppose that $\lim _{n=\infty} s_{n}=s$ and write $s_{n}=s+\epsilon_{n}$. Then we have

$$
t_{r}=s \sum_{k=0}^{\infty} c_{r k}+\sum_{k=0}^{\infty} c_{r k} \epsilon_{k}
$$

whence it follows at once that $\lim _{r=\infty} t_{r}=s$.

Thus we have the following result:

Theorem IX. A sufficient condition that the definition associated with (22) shall be regular is that

1) $\lim c_{r k}=0$ for every $k$;

2) the series $c_{r 0}+c_{r 1}+\cdots$ shall converge absolutely and its sum shall approach unity as $r$ approaches infinity;

3) for every $r$ the sum of the series $\left|c_{r 0}\right|+\left|c_{r 1}\right|+\cdots$ shall be less than a quantity $M$ independent of $r$.

The first two of these conditions are necessary for the regularity of the definition.*

In the case when the quantities $c_{r k}$ are non-negative and real, conditions 1) and 2) are necessary and sufficient for the regularity of the definition.

Let us consider similarly the definition in accordance with which series (1) is said to have the sum $t$ in case $\lim _{r=\infty} t_{r}$ exists and has the finite value $t$, where

$$
t_{r}=\sum_{k=0}^{\infty} a_{r k} u_{k}
$$

the $a_{r j}$ being (real or complex) constants.

In order that the definition shall be regular it is necessary in the first place that $\lim _{r=\infty} t_{r}$ shall be unity when $u_{k}=1$ and $u_{i}=0$ for $i \neq k$. Hence we must have $\lim _{r=\infty} a_{r k}=1$ for

* There is an obvious incompleteness about this theorem and the following one, presented here in the form in which I gave them in the lecture at Chicago. It was my intention to complete them before publication. But on the day following my lecture Professor T. H. Hildebrandt read a paper before the American Mathematical Society at Chicago giving the theorems in all their completeness and in elegant form, his results having been obtained before he knew of mine. Consequently I am leaving to him the duty of making known these desired results in their complete and satisfactory form. 
every $k$. In what follows we assume that this condition is satisfied.

Again, in order that $t_{r}$ shall be defined whenever (1) is convergent it is necessary (and sufficient) that the limit,

$$
\lim _{n=\infty} \sum_{k=0}^{n} a_{r k} u_{k},
$$

shall exist and be finite for every converging series (1). We may write

$$
\begin{aligned}
y_{r n}=\sum_{k=0}^{n} a_{r k} u_{k} & =\sum_{k=1}^{n} a_{r k}\left(s_{k}-s_{k-1}\right)+a_{r 0} s_{0} \\
& =\sum_{k=0}^{n-1} s_{k}\left(a_{r k}-a_{r, k+1}\right)+a_{r n} s_{n} .
\end{aligned}
$$

It follows now from the corollary to Theorem I that $\lim _{n=\infty} y_{r n}$ will exist and be finite for every converging series (1) when and only when a constant $M$, independent of $r$, exists such that

$$
\left|a_{r n}\right|+\sum_{k=1}^{n}\left|a_{r k}-a_{r, k+1}\right|<M \text { for every } r ;
$$

and hence when and only when the series*

$$
\sum_{k=1}^{\infty}\left|a_{r k}-a_{r, k+1}\right|
$$

is convergent. This implies, in particular, that $\lim _{n=\infty} a_{r n}$ exists and is finite.

If in addition to the foregoing limitations on the coefficients $a_{r j}$ they are further restricted by the requirement that a constant $M_{1}$ shall exist, independent of $r$, such that the sum of the series in (26) shall be less than $M_{1}$ for every $r$, it is easy to show that our definition is regular. For, in view of (25) we have

$$
\begin{aligned}
t=\lim _{r=\infty} t_{r}= & \lim _{r=\infty}\left[\sum_{k=0}^{\infty} s_{k}\left(a_{r k}-a_{r, k+1}\right)+s \lim _{n=\infty} a_{r n}\right] \\
=\lim _{r=\infty}\left[\sum_{k=0}^{\infty} s\left(a_{r k}-a_{r, k+1}\right)+\right. & s \lim _{n=\infty} a_{r n} \\
& \left.+\sum_{k=0}^{\infty} \epsilon_{k}\left(a_{r k}-a_{r, k+1}\right)\right],
\end{aligned}
$$

* This result is obtained by Kojima, l.c., p. 305. 
where $s+\epsilon_{k}$ is written for $s_{k}$. Hence

$$
t=s+\lim _{r=\infty} \sum_{k=0}^{\infty} \epsilon_{k}\left(a_{r k}-a_{r, k+1}\right),
$$

whence it follows at once that $t=s$.

Hence we have the following theorem:*

Theorem X. A sufficient condition that the definition associated with (23) shall be regular is that

1) $\lim _{r=\infty} a_{r k}=1$ for every $k$;

2) the series $\left|a_{r 0}-a_{r 1}\right|+\left|a_{r 1}-a_{r 2}\right|+\ldots$ shall converge;

3) a constant $M$, independent of $r$, shall exist such that the sum of the series in 2) shall be less than $M$ for every $r$.

Moreover, the first two of these conditions are necessary. $\dagger$

In the case when the quantities $a_{r k}-a_{r, k+1}$ are all real and not negative, or all real and not positive, it is obvious that condition 3) is implied by conditions 1) and 2); and hence in either of the conditions named 1) and 2) are necessary and sufficient for the regularity of the definition.

The regularity of Borel's exponential definition follows at once from Theorem IX. For, we have

$$
c_{r k}=\frac{r^{k}}{k !} e^{-r}
$$

so that conditions 1), 2), 3) of the theorem are satisfied. $\ddagger$

By writing $r x=r-1$ LeRoy's definition (15) may be thrown into the form

$$
t=\lim _{x=1-0} \sum_{k=0}^{\infty} \frac{\Gamma(k x+1)}{\Gamma(k+1)} u_{k}=\lim _{r=\infty} \sum_{k=0}^{\infty} \frac{\Gamma(k-k / r+1)}{\Gamma(k+1)} u_{k} .
$$

If we put

$$
a_{r k}=\frac{\Gamma(k-k / r+1)}{\Gamma(k+1)}=\left(1-\frac{1}{r}\right) \frac{\Gamma(k-k / r)}{\Gamma(k)}
$$

and employ the asymptotic properties of the gamma function

* Certain related but different results are due to C. N. Moore, Transactions Amer. Math. Society, vol. 8 (1907), pp. 299-330. The same author has also given similar theorems for double series, ibid., vol. 14 (1913), pp. 73-104.

$\dagger$ See the footnote to the preceding theorem.

$\ddagger$ For additional theorems concerning Borel's exponential method of summation see Hardy and Littlewood, Palermo Rendiconti, vol. 41 (1916), pp. 36-53. 
it is easy to show that the conditions of Theorem $\mathrm{X}$ are satisfied and hence that the definition in consideration is regular.

According to Borel's integral method of summation we take for the sum of (1) the quantity,

$$
t=\int_{0}^{\infty} e^{-a}\left(\sum_{k=0}^{\infty} \frac{a^{k}}{k !} u_{k}\right) d a,
$$

where it is assumed that series (1) is such that the infinite series appearing in this expression converges for every value of $a$. This may be written in the form

$$
\begin{aligned}
t & =\lim _{r=\infty} \int_{0}^{r} e^{-a}\left(\sum_{k=0}^{\infty} \frac{a^{k}}{k !} u_{k}\right) d a \\
& =\lim _{r=\infty} \lim _{n=\infty} \sum_{k=0}^{n}\left[\int_{0}^{r} e^{-a} \frac{a^{k}}{k !} d a\right] u_{k} .
\end{aligned}
$$

Hence, if we take

$$
a_{r k}=\int_{0}^{r} e^{-a} \frac{a^{k}}{k !} d a,
$$

we exhibit the Borel integral definition as an element of the class of definitions by the method of mean values with infinite reference. Since

$$
a_{r k}-a_{r, k+1}=e^{-r} \frac{r^{k+1}}{(k+1) !},
$$

as one sees readily through integrating by parts the integral defining $a_{r, k+1}$, it is easy to show that the hypotheses of theorem $\mathrm{X}$ are satisfied; and hence that the Borel integral definition is regular.

A comparison of Theorems IX and X with Theorems I and II brings out the fact (already alluded to in the footnotes) that the former are not in altogether the same satisfactory form as the latter; they are completed by the (as yet unpublished) work of Hildebrandt. So far as I am aware no general theorems yet exist concerning the mutual consistency of definitions by the method of mean values with infinite reference nor concerning the equivalence of such definitions (see related matters in $\$ \S 3$ and 4). We may propose these as two fundamental problems, or perhaps as two aspects of the same fundamental problem, in the theory of summable series. Other considerations analogous to those of $\$ 5$ still await de- 
velopment for the case of definitions by the method of mean values with infinite reference.

\section{\$7. Definitions of Sum by Means of Integrals.}

The theory of Borel's definition of sum by means of an integral has been so well expounded in Borel's Séries divergentes and Bromwich's Infinite Series that we need to give here only a brief summary of the main results.* We confine attention to the case of absolute summability. $\dagger$

If one has a polynomial function $P(u, v, w, \cdots)$ of a finite number of variables $u, v, w, \cdots$, the coefficients being numerical, and if one replaces $u, v, w, \cdots$ by absolutely summable series and combines the result according to the usual rules of operating with convergent series one obtains an absolutely summable series whose sum is equal to the numerical value obtained by replacing $u, v, w, \cdots$ in $P$ by the sums of the corresponding series (Borel, l. c., page 108). $\ddagger$

Denote by $u, v, w$ power series

$$
u=\sum_{n=0}^{\infty} u_{n} x^{n}, \quad v=\sum_{n=0}^{\infty} v_{n} x^{n}, \quad w=\sum_{n=0}^{\infty} w_{n} x^{n},
$$

and suppose that these are absolutely summable for $x=x_{0}$. Let

$$
P\left(u, v, w, u^{\prime}, v^{\prime}, w^{\prime}, \cdots, u^{(\lambda)}, v^{(\lambda)}, w^{(\lambda)}, x\right)
$$

denote a polynomial in $u, v, w$ and their derivatives up to order $\lambda$ inclusive, the coefficients of which are series in integral powers of $x$ whose radius of convergence is greater than $\left|x_{0}\right|$. If in $P$ one replaces $u, v, w$ by the corresponding series and performs the indicated operations as if the series were convergent, one obtains a series $S$ which is absolutely summable for each value of $x$ on the straight line from 0 to $x_{0}$ (exclusive of $x_{0}$ itself) and which defines an analytic function $F$ which is regular in the interior of a circle of which the line joining 0 and $x_{0}$ is a diameter. This analytic function is precisely that

\footnotetext{
* In the preceding section we saw that Borel's definition of summability (but not of absolute summability) is regular.

† Borel's statement (1. c., p. 100) that every convergent series is absolutely summable is incorrect, as has been pointed out by Hardy, Quar Journal of Math., vol. 35 (1903), pp. 25-28. But every absolutely convergent series is absolutely summable.

$\ddagger$ Related results for summable series (not absolutely summable) are given by Hardy, l. c., p. 43.
} 
which $P$ becomes when in $P$ one replaces $u, v, w$ not by the series but by the corresponding analytic functions. Moreover, $F$ is identically zero when and only when the series $S$ has all its coefficients zero, that is to say, when and only when the series $u, v, w$ formally satisfy the relation

$$
P\left(u, v, w, u^{\prime}, \cdots, w^{(\lambda)}, x\right)=0 .
$$

In this case the corresponding analytic functions $u, v, w$ also satisfy this relation (Borel, l. c., page 114).

If the foregoing theorem is applied to the case of the differential equation

$$
f\left(x, y, y^{\prime}, \cdots, y^{(\lambda)}\right)=0,
$$

where $f$ is analytic in $x$ at $x=0$ and algebraic in $y$ and its derivatives, it results at once that, if an absolutely summable series $y$ formally satisfies the differential equation, the analytic function defined by the Borel integral sum of this series is a solution of the differential equation (Borel, l. c., page 115).

Suppose that the power series

$$
\varphi(x)=u_{0}+u_{1} x+u_{2} x^{2}+\cdots .
$$

has a finite (non-zero) radius of convergence. Let $l$ denote a line through a singularity of the function defined by the power series and perpendicular to the radius vector from zero to this singularity. Let $P$ denote the "polygon" which contains in its interior the point zero and extends from zero in every given direction to the nearest of these lines $l$ in such direction. Then the series $\varphi(x)$ is absolutely summable for points in the interior of $P$; it is not absolutely summable at any point exterior to $P$; on the boundary of $P$ it may or may not be absolutely summable (Borel, l. c., page 128).*

Through use of the foregoing result and tests for the absolute summability of series one is able to treat certain phases of the problem as to the position of the singularities of a function defined by a power series (Borel, l. c., page 136).*

Borel's integral method of summation is thus seen to yield an important contribution (among others) to the fundamental

* The method of analytic continuation suggested by the results in these two paragraphs may be extended by the use of a definition of summability based on the function $\varphi(a)=e^{a^{k}}$ instead of on the function $\varphi(a)=e^{a}$ (Borel, l. c., p. $129 \mathrm{ff}$.). The "polygon" of summability is replaced by a certain curvilinear figure. See also a paper by E. Lindelöf, Bull. Soc. Math. France, vol. 29 (1901), pp. 157-160. 
problem of deducing the properties of an analytic function from the properties of its Taylor development.* Contributions to the study of this problem have been made by many authors from diverse points of view. One of the most important of these in connection with the subject of summability is a memoir by LeRoy. $\dagger$

Let us consider the divergent series

Put

$$
\sum_{n=0}^{\infty} \alpha_{n} z^{n} .
$$

$$
\alpha_{n}=\Gamma(p n+1) a_{n}
$$

and denote by $F(z)$ the function

$$
F(z)=\sum_{n=0}^{\infty} a_{n} z^{n} .
$$

Suppose that the radius of the circle of convergence of the last series is finite (and different from zero) and that the circle of convergence is not a natural boundary of the function defined by it. Then for the sum $f(z)$ of the first series LeRoy (l. c., page 416) writes

$$
f(z)=\sum_{n=0}^{\infty} \alpha_{n} z^{n}=\frac{1}{p} \int_{0}^{\infty} e^{-x^{1 / p}} x^{-1+1 / p} F(z x) d x,
$$

provided that the last integral is convergent. For $p=1$ this reduces to Borel's definition. Under appropriate broad conditions such summable divergent series as are here introduced are amenable to the usual methods of computation employed in the case of convergent series. The theory has applications to differential equations and analytic continuation of the same essential character as those of the special case in which Borel's definition is sufficiently far-reaching. We must refer the reader to LeRoy's memoir for a fuller account of his important investigations in connection with this and other

\footnotetext{
* Conversely, one may think of any method of analytic continuation as a method for the summation of divergent series (Borel, 1. c., p. 120). Note how LeRoy (see next footnote) has been led in this way to new definitions of sum. See especially pp. $405 \mathrm{ff}$. of his paper.

$\dagger$ Toulouse Annales, ser. 2 , vol. 2 (1900), pp. 317-430. See also the work of Servant (referred to at the end of $\$ 1$ above).
} 
definitions of summability. See also his notes in Paris Comptes Rendus for 1898, 1899, 1900.*

Reference may also be made to extensions of Borel's method by Cunningham $\dagger$ and by Buhl. $\ddagger$ Compare also the method of Barnes (see reference near end of $\S 1$ ).

\section{\$8. General Requirements Which Should be Met in Any Defin- ition of Sum of an Infinite Series.}

In §5 we have considered some general requirements which should be met by every definition of sum of an infinite series. It appears that a great many definitions, not all mutually consistent, meet all these requirements. In view of this fact and with a desire to introduce greater uniformity into the general theory of summability, some authors have felt that certain further general restrictions are distinctly to be desired. Thus W. O. Mendenhall§ and W. B. Ford $\|$ have insisted on the desirability of imposing the so-called boundary value condition. Thus these authors propose to confine attention to those series (1) for which the corresponding power series

$$
f(x)=\sum_{n=0}^{\infty} u_{n} x^{n}
$$

has a radius of convergence equal to unity $\uparrow$ and then to agree to retain those definitions alone for which the sum $s$ of (1), when existent, satisfies the relation**

\footnotetext{
* Besides the references already given see also Ricotti, Giornale de Matematiche, vol. 48 (1910), pp. 79-111, for a treatment of the methods of Borel and LeRoy; Maillet, Annales Ecole Norm. Sup., ser. 3, vol. 20 (1903), pp. 487-518; Van Vleck, Boston Colloquium, 1903, pp. 92-107.

$\dagger$ Proc. London Math. Society, ser. 2, vol. 3 (1905), pp. 157-169.

$¥$ Bulletin des Sciences mathématiques, vol. 42 (1907), pp. 340-346.

$\$$ Michigan dissertation, 1911. This dissertation has not been published. Through the kind response of the author to a request of mine I have had the opportunity to examine a manuscript copy of it. Its more important results are to be found in Ford's book on divergent series.

\|| Ford, Studies on Divergent Series and Summability, pp. $82 \mathrm{ff}$. Also in his address (December, 1917) as retiring chairman of the Chicago Section of the American Mathematical Society, this Bulletin, vol. 25 (1918), pp. 1-15.

If The restriction to such series appears to be particularly unfortunate in view of the important applications of the theory of summability to power series with zero radius of convergence.

** The demand here essentially is that we shall restrict attention to certain of those definitions of sum which are mutually consistent with a single given definition, namely, that in which series (1) is said to have the
} 


$$
s=\lim _{x=1-0} f(x) \text {. }
$$

An earlier treatment of the general considerations involved in a restriction of this sort is to be found in LeRoy's memoir (cited in \$7). He takes a more general point of view than Mendenhall and Ford in that he allows the radius of convergence of the power series to be less than 1 and considers the question of assigning to series (1) the sum $f(1)$, where an element of $f(x)$ is defined as above. He also discusses the problem for the case when the radius of convergence of the power series is zero.

In view of the fact that convergent power series are a much restricted class of infinite series and that the theory of summability has made significant and important conquests in the theory of expansions in orthogonal functions (including Fourier series), in the theory of Dirichlet series and factorial series, and promises important applications to the more general expansion problems arising in the theory of difference equations, such a restriction as that desired by Mendenhall and Ford should be insisted upon only for the most cogent reasons. Furthermore one of the far-reaching applications of the theory of summability is to the case of descending power series which diverge for every value of the variable, an application which leads to consequences of large importance in the theory of differential and difference equations. Hence this class of series must not be ruled out; moreover, it is difficult to see why a definition which is to be used in the case of such series must of necessity satisfy the boundary value condition. Thus it appears to me that no satisfactory reasons have been advanced for this restriction proposed by Mendenhall and Ford and consequently that there is not yet any sufficient ground for confining attention to definitions satisfying the boundary value condition. Those which do satisfy it doubtless form an important class having a well-defined usefulness in the study of analytic functions; but there seems not yet to be any valid reason for supposing that other classes are not also important.

sum $s$ when the limit

$$
\lim _{x=1-0}\left(u_{0}+u_{1} x+u_{2} x^{2}+\ldots\right)
$$

exists and has the finite value $s$; and that we shall reject every definition which assigns a sum to a series (1) to which this last definition does not assign a sum. 
For the case of only a few definitions has it yet been determined whether the so-called boundary value condition is satisfied. For these consult the work of Mendenhall and Ford.

The other extreme as regards the matter of limitation upon the definition of summability or freedom in this respect has not failed to be represented in the literature. Thus Hardy* has formulated a principle which Bromwich (Infinite Series, pages 267-8) states in the following words: "If two limiting processes, performed in a definite order on a function of two variables, lead to a definite value $X$, but, when performed in the reverse order, lead to a meaningless expression $Y$, we may agree to interpret $Y$ as meaning $X$." The property implied in this principle certainly belongs to many of the current definitions of sum; but it does not appear to have been a useful guide in the formulation of any of the important special definitions. It seems to allow too great a variety of possibilities to provide anything of marked value in the way of specific definitions. It is useful, however, as a unifying principle.

\section{§9. Applications to the Theory of Dirichlet Series.}

From Theorem VII it follows that Cesàro's methods of summation are limited in a way which forbids their application to the problem of the analytical continuation of a function defined by a power series; but they have been of the greatest use in the study of the function on the circle of convergence of the power series which represents it. Owing to the delicate character of the convergence of a Dirichlet series it is natural to suppose that Cesàro's methods would find wider application here than in the theory of power series whose character of convergence is much more crude. The first applications of this sort were made independently by Bohr and M. Riesz, who showed that the region of Cesàro summability of a Dirichlet series may be greater than its region of convergence, so that there exist regions of summability in which the series diverges while, nevertheless, Cesàro's means afford the ana-

*Quar. Journal Math., vol. 35 (1904), pp. 22-47. See also Hardy and Chapman, ibid., vol. 42 (1911), pp. 181-215; Chapman, ibid., vol. 43 (1911), pp. 1-52. In the last paper Chapman treats a generalization of Hardy's principle. 
lytical continuation of the function defined by the series within its region of convergence.*

Although Cesàro's methods thus have an important application in the theory of Dirichlet series it appeared from the investigations of Riesz that a certain disadvantage still existed which might be overcome by the introduction of a new type of definition which should maintain certain features belonging to the definitions of Cesàro. Thus Riesz was led to introduce his definition by "typical means," as given in $\$ 1$ in connection with relation (12).

In the case when $\lambda_{n}=n$ it has been shown by Riesz $\dagger$ that his definitions, for varying $r$, are equivalent to those of Cesàro, Knopp, and Chapman, when in each case the same value of $r$ is taken.

An excellent account of this method of summation and of its application to the theory of Dirichlet series has been given by Hardy and Riesz. $\ddagger$ For this reason we shall not attempt an exposition of the matter. We state merely a few of the leading properties of the definition as developed by these authors.

The definitions of Riesz are regular. If (1) is summable $(R \lambda r)$ to the sum $s$, it is summable $\left(R \lambda r^{\prime}\right)$ to the same sum $s$, for every $r^{\prime}$ greater than $r$. If (1) is summable $(R l r)$, where $l_{n}=e^{\lambda n}$, then it is summable $(R \lambda r)$ to the same sum. If (1) is summable $(R \lambda r)$, where $\lambda_{n}=n$, it is also summable $(R \lambda r)$ to the same sum where $\lambda_{n}=\log n$. If $\mu$ is any logarithmicoexponential function of $\lambda$, and if (1) is summable $(R \lambda r)$ then it is summable $(R \mu r)$. Speaking roughly, we may say (in view of the last result) that the efficacy of the method $(R \lambda r)$ increases as the rate of increase of the function $\lambda$ decreases. $\S$

* Borel's exponential method of summation is also applicable to Dirichlet series; see Hardy, Proc. London Math. Society, ser. 2, vol. 8 (1909), pp. 277-294. See also Hardy's paper on the so-called Abel's method of summation as applied to Dirichlet series, Quar. Journal of Math. vol. 47 (1916), pp. 176-192.

† Paris Comptes Rendus, vol. 152 (1911), pp. 1651-1654.

‡ "The General Theory of Dirichlet Series," Cambridge University Press, 1915. This monograph should also be consulted for treatment of other matters relating to the summability of Dirichlet series.

$\S$ We may refer to the following papers which appeared later than the Hardy-Riesz monograph:

Nalli, Palermo Rendiconti, vol. 40 (1915), pp. 44-70; 42 (1917), pp. 61-72.

Hardy, Paris Comptes Rendus, vol. 162 (1916), pp. 463-466.

Hardy, Quar. Journal Math., vol. 47 (1916), pp. 176-192.

Hardy, Proc. London Math. Society, ser. 2, vol. 15 (1916), pp. 72-88. 
\$10. Applications to the Expansion Problems Arising in the Theory of Difference Equations.

This account of the general aspects of the theory of summability we shall bring to a close by indicating briefly an important application the development of which lies mostly in the future. In several memoirs* which I have presented to the Society in the last two or three years I have given an outline of the main features of what appears to me to be the central expansion problem in the theory of difference equations. This problem has to do with series of the form $\dagger$

$$
\Omega(x)=\sum_{n=0}^{\infty} c_{n} \frac{g(x+n)}{g(x)}
$$

where the quantities $c_{n}$ are independent of $x$ and the function $g(x)$ is restricted mainly as to its analytic and asymptotic character in a certain sector $V$ including in its interior the positive axis of reals. The asymptotic form is as follows:

$$
g(x) \sim x^{P(x)} e^{Q(x)}\left(1+\frac{a_{1}}{x}+\frac{a_{2}}{x^{2}}+\cdots\right),
$$

where $P(x)$ and $Q(x)$ are polynomials and the $a$ 's are constants. It is assumed that $g(x)$ is analytic in $V$ when $|x|$ is sufficiently large but finite.

The most important cases (and the ones arising most naturally from the theory of linear difference equations) are those in which $P(x)$ is a linear function of $x$ and $Q(x)$ is a linear function or a constant. Certain special functions $g(x)$ belonging to the class thus defined give rise (see memoir II) to factorial series and to some generalizations of them which play the fundamental rôle in certain important recent investigations (see references in memoir II).

* These memoirs will be referred to by the numbers in the following list:

I. Transactions Amer. Math. Society, vol. 17 (1916), pp. 207-232.

II. Bulletin Amer. Math. Society, vol. 23 (1917), pp. 407-425.

III. Amer. Journal of Math., vol. 39 (1917), pp. 385-403.

IV. Amer. Journal of Math., vol. 40 (1918), pp. 113-126.

$\dagger$ A similar problem arises for series of the form

$$
\sum_{n=0}^{\infty} c_{n} \frac{g(n x)}{g(x)},
$$

where $g(x)$ has asymptotic properties similar to those defined in the text. These series are now being investigated by L. L. Steimley, a student in the University of Illinois. 
So far the question of summability of series $\Omega(x)$ has been treated only for the case of factorial series; and the methods of Cesàro alone have been used for this purpose. Landau* has shown that the factorial series and the Dirichlet series

$$
\sum_{s=0}^{\infty} \frac{a_{s} s !}{x(x+1) \cdots(x+s)}, \quad \sum_{s=1}^{\infty} \frac{a_{s}}{s^{x}}
$$

have the same points of convergence (except for $x=0,-1$, $-2, \ldots$. .). The interior of the region of convergence of each series is the portion of a plane to the right of a line $R(x)=\lambda$, where $\lambda$ is an appropriately determined constant and $R(x)$ stands for the real part of $x$. Bohr $\dagger$ has established the existence of a sequence of real numbers $\lambda_{1}, \lambda_{2}, \lambda_{3}, \ldots\left(\lambda_{1} \geqq \lambda_{2}\right.$ $\geqq \lambda_{3} \geqq$...) such that each of the two foregoing series is summable $(C r)$ in a half-plane $R(x)>\lambda_{r}(r=1,2,3, \ldots)$ but not for $R(x)<\lambda_{r}$. As $n$ approaches infinity $\lambda_{n}$ approaches a limit $\bar{\lambda}$. Bohr (l. c.) has shown that the line $R(x)=\bar{\lambda}$ plays a fundamental rôle for functions defined by the Dirichlet series. Nörlund $\ddagger$ has pointed out that the number $\bar{\lambda}$ does not enjoy a similar property for the factorial series; this raises the question as to whether other methods of summation may do for the factorial series what the methods of Cesàro do for Dirichlet series.

The function $f(x)$ defined by the foregoing factorial series has an asymptotic representation§ (in general divergent) of the form

$$
f(x)=\frac{A_{0}}{x}+\frac{A_{1}}{x^{2}}+\frac{A_{2}}{x^{3}}+\cdots .
$$

This series is summable by the exponential method of Borel

* Münchener Sitzungsberichte, vol. 36 (1906), pp. 151-218.

$\dagger$ Göttinger Nachrichten, 1904, pp. 247-262. Bohr states also several important properties of the regions of summability of different orders. $\mathrm{He}$ also derives similar results for the case of series of binomial coefficients. $\ddagger$ Paris Comptes Rendus, vol. 158 (1914), pp. 1325-1328.

\$ See Nielsen, Annales Ecole Norm. Sup., ser. 3, vol. 2 (1904), pp. 449458. See also the treatment of a more general problem in my memoirs III and IV.

|| Nörlund, l. c.; Paris Comptes Rendus, vol. 158 (1914), pp. 1252-1253; Acta Mathematica, vol. 37 (1914), pp. 327-387. A certain natural extension of factorial series is treated here, the theory still being contained in that of the general series $\Omega(x)$.

Compare also in this connection, Pincherle, R. Accad. L. Rend., ser. 5, vol. 13 (1904), pp. 513-519, and my memoir IV already cited. 
and the sum obtained by this method is $f(x)$ :

where

$$
f(x)=\int_{0}^{\infty} e^{-x t} F(t) d t,
$$

$$
F(t)=A_{0}+\frac{A_{1}}{1 !} x+\frac{A_{2}}{2 !} x^{2}+\cdots .
$$

A number $l$ exists such that this integral converges when $R(x)>l$ and diverges when $R(x)<l$. This number $l$ plays a fundamental rôle as regards the properties of the function $f(x)$ defined by the factorial series.

We have here in a special case two aspects of the general theory of summability of the series $\Omega(x)$, a theory of importance the development of which will lead to significant extensions of our knowledge of one of the most fundamental expansion problems in analysis. Early in 1917, Mr. Charles F. Green, a student at the University of Illinois, was beginning work upon this subject, looking towards a doctor's dissertation; but his labor has been interrupted by more pressing duties and he is now engaged as a pilot in the aviation service with the American Army in France.

UNIVERSITY OF IlLINOIS.

\section{ON THE PROBLEM OF THE RESISTANCE INTEGRAL.}

BY PROFESSOR TSURUICHI HAYASHI.

THE problem of minimizing the resistance integral seems to be of three main varieties.

1. Newton's problem:*

To get a solid of revolution formed by revolving a curve passing through two given points about an axis which shall experience a minimum resistance when it moves through a fluid in the direction of its axis.

The solution is the well-known transcendental curve.

* Philosophiæ Naturalis Principia Mathematica, 1687, Book 2, Section 7, Prop. 34, Scholium. 